\title{
Upregulation and redistribution of integrin $\alpha 6 \beta 4$ expression occurs at an early stage in pancreatic adenocarcinoma progression
}

\author{
Zobeida Cruz-Monserrate ${ }^{1}$, Suimin Qiu $^{2}$, B Mark Evers ${ }^{1,3}$ and Kathleen L O’Connor ${ }^{1,3}$ \\ ${ }^{1}$ Sealy Center for Cancer Cell Biology, University of Texas Medical Branch, Galveston, TX, USA; ${ }^{2}$ Department \\ of Pathology, University of Texas Medical Branch, Galveston, TX, USA and ${ }^{3}$ Department of Surgery, \\ University of Texas Medical Branch, Galveston, TX, USA
}

\begin{abstract}
Pancreatic adenocarcinomas are highly invasive cancers for reasons that are currently unclear. Here we sought to determine if the proinvasive integrin $\alpha 6 \beta 4$ may be related to pancreatic adenocarcinoma tumor progression. Expression of integrin $\alpha 6 \beta 4$ was analyzed via immunohistochemistry for the $\beta 4$ subunit in normal pancreas, pancreatic intraepithelial neoplasia (PanIN) lesions, pancreatic adenocarcinomas and chronic pancreatitis. In normal pancreatic ducts, integrin $\alpha 6 \beta 4$ was noted only at the cell's basal interface with the basement membrane. In pancreatic adenocarcinomas, 92\% (104/113) demonstrated overexpression of integrin $\alpha 6 \beta 4$ and altered localization to the cytoplasm and membranous regions. This pattern of expression was observed in all PanIN lesions as early as PanIN-1A, and was evident in lesions that were juxtapositioned to normal epithelium. In contrast, $93 \%$ (13/14) of chronic pancreatitis samples resembled the staining pattern of normal pancreas. When cancer was present in areas of chronic pancreatitis, this altered expression of $\alpha 6 \beta 4$ integrin identified the cancer. We conclude that integrin $\alpha 6 \beta 4$ is expressed only on the basal surface of ductal cells in normal pancreas and chronic pancreatitis. During pancreatic adenocarcinoma progression, the $\alpha 6 \beta 4$ integrin is dramatically overexpressed and displays altered localization at the earliest stages of PanIN, thus representing an early event in pancreatic adenocarcinoma progression.
\end{abstract}

Modern Pathology (2007) 20, 656-667; doi:10.1038/modpathol.3800782; published online 13 April 2007

Keywords: pancreatic intraepithelial neoplasia (PanINs); immunohistochemistry; precursor lesion; integrins; pancreatic cancer; chronic pancreatitis

Pancreatic carcinoma is the fourth leading cause of cancer death in the US and has the highest death to incidence ratio of all cancers. ${ }^{1}$ Poor prognosis of pancreatic cancer patients relates to a high incidence of tumor cell invasion and metastasis. Treatment options are limited and patients succumb to the disease shortly after diagnosis unless eligible for tumor resection. ${ }^{2}$ Of those patients that undergo resection, only $15-20 \%$ will survive to 5 years. ${ }^{1}$

Over $90 \%$ of pancreatic cancers are adenocarcinomas that are thought to arise from proliferative premalignant pancreatic intraepithelial neoplasia (PanIN) of the ductal epithelium. PanIN lesions start as low cuboidal epithelial cells that become columnar due to increased mucin production. Cells

Correspondence: Dr KL O’Connor, PhD, Department of Surgery, University of Texas Medical Branch, 301 University Blvd., Galveston, TX 77555-0525, USA.

E-mail: kloconno@utmb.edu

Received 9 December 2006; revised 5 March 2007; accepted 7 March 2007; published online 13 April 2007 then present with nuclear atypia and enhanced proliferation, which lead to luminal shedding and/ or invasion into the stroma. ${ }^{3}$ These morphological alterations correlate with increased genetic abnormalities such as the activation of K-ras, loss of tumor suppressors (eg p16, p53 and DPC4) and upregulation of telomerases. ${ }^{4}$ Although PanIN-1A and PanIN1B are considered early cancer precursor lesions, molecular studies have shown that PanIN-2 and PanIN-3 lesions represent a distinct step toward invasive carcinoma. ${ }^{5}$ Moreover, PanIN lesions can be found in patients with chronic pancreatitis ${ }^{6}$ and these patients have an increased risk of developing pancreatic cancer. ${ }^{7,8}$

Infiltrating duct-like and tubular structures embedded in a highly desmoplastic stroma are characteristic of pancreatic cancers but also are features of chronic pancreatitis. The overlap in the clinical presentation and histopathological features between chronic pancreatitis and pancreatic cancer can lead to confusion in the diagnosis and management of both diseases. ${ }^{9}$ While histological parameters are 
useful, more specific markers are needed to detect pancreatic cancer at an early stage and distinguish pancreatic cancer from chronic pancreatitis. The quest for such markers has led to the use of high throughput technology such as genetic profiling and proteomics. Recent studies that included the use of genetic profiling and immunohistochemistry suggested that the integrin $\alpha 6 \beta 4$ is selectively overexpressed in pancreatic adenocarcinomas, ${ }^{10-13}$ thus providing evidence of the potential role of this integrin in pancreatic cancer progression.

Integrins are receptors for extracellular matrices that transmit mechanical and biochemical signals to regulate cellular functions including survival, proliferation, motility, transcription and protein translation. They consist of two type I transmembrane $\alpha$ and $\beta$ subunits that associate in various combinations to form at least 25 receptors. ${ }^{14}$ Integrin adhesion to extracellular matrices and signaling have been associated with tumor development, invasion, and metastasis. ${ }^{15}$ In particular, the $\alpha 6 \beta 4$ integrin is increased in several types of invasive and metastatic carcinomas with increased levels of expression correlating with a highly invasive and motile phenotype ${ }^{16}$ as well as reduced patient survival. ${ }^{17}$ The $\beta 4$ integrin subunit is unique in that its cytoplasmic domain is 1000 amino acids longer than that of other integrins and its extracellular domain associates exclusively with the $\alpha 6$ integrin subunit. ${ }^{18}$ One function of the $\alpha 6 \beta 4$ integrin is to maintain the structure and integrity of epithelia through the formation of hemidesmosomes. ${ }^{19} \mathrm{In}$ cancers, integrin $\alpha 6 \beta 4$ is released from the hemidesmosomes in epithelial cells and associates with the actin cytoskeleton. ${ }^{20}$ When released from hemidesmosomes, the $\alpha 6 \beta 4$ integrin contributes to an invasive phenotype by signaling to molecules such as phosphoinositol-3-kinase and cooperating with growth factors. ${ }^{21}$

The strong link between the $\alpha 6 \beta 4$ integrin and an invasive phenotype coupled with gene array data that shows upregulation of this integrin in pancreatic adenocarcinomas prompted us to investigate if the $\alpha 6 \beta 4$ integrin is associated with the aggressiveness and progression of pancreatic cancers. Therefore, the goals of this study were: (1) to compare the localization and expression levels of integrin $\alpha 6 \beta 4$ in normal pancreas, chronic pancreatitis and pancreatic adenocarcinomas and (2) to establish if integrin $\alpha 6 \beta 4$ expression and localization is associated with pancreatic cancer tumor progression.

\section{Materials and methods}

\section{Tissue Specimens}

Paraffin-embedded archival tissues from patients with histological normal pancreas $(n=4$; two cases underwent tumor resections for other gastrointestinal cancers in which the pancreas was free of tumor, one suspected pancreatic cancer case was found to be normal after resection was performed and one case was a benign pancreatic tumor which contained ample normal pancreatic tissue for analysis), pancreatic adenocarcinomas $(n=20)$, and chronic pancreatitis $(n=14)$ were obtained from the UTMB Surgical Pathology Department using an IRB-approved protocol. Of the 20 pancreatic adenocarcinoma cases, 12 cases included regions of normal uninvolved tissue and 15 cases presented with both pancreatic adenocarcinoma and chronic pancreatitis. In most of these cases, multiple tissue specimens were used in the analysis with tissue size varying from a few $\mathrm{mm}$ to 2 inches. In addition, three pancreatic cancer tissue microarrays embedded in paraffin were used: AccuMax Array A207 (II) (1.5 mm spots, two spots per case), AccuMax Array A207 (III) (1 mm spots, two spots per case) (ISU ABXIS Co., Seoul, South Korea) and the PA801 array (1.5 mm spots, one spot per case) (US Biomax, Inc., Rockville, MD, USA). The cases included in our study analyzes 93 pancreatic adenocarcinomas that came from these tissue arrays, for which 23 cases included normal uninvolved tissue. Pathology of all specimens was determined based on the histology of serial sections stained with hematoxylin and eosin (H\&E) using standard criteria.

Overall, our study utilized 131 cases, which included four cases with histological normal pancreas, 14 chronic pancreatitis and 113 pancreatic adenocarcinomas (which included 35 cases with regions of normal uninvolved tissue and 15 cases for which the specimens presented with pancreatic adenocarcinoma and chronic pancreatitis). The average age of all cases was 57 (range 22-78) including 43 female and 88 male subjects.

\section{Immunohistochemical Staining}

Tissue sections ( $4 \mu \mathrm{m})$ and tissue microarrays ( $5 \mu \mathrm{m})$ were deparaffinized with xylene and rehydrated in decreasing concentrations of alcohol. Endogenous peroxidase activity was blocked by incubating slides with $3 \%$ hydrogen peroxide in $1 \times$ Dulbecco's phosphate-buffer saline (DPBS; pH 7.4) (Gibco, Carlsbad, CA, USA) for $10 \mathrm{~min}$. After rinsing with water, antigen retrieval was performed by placing slides inside a container filled with $1 \times$ Dako Target Retrieval Solution (S1699) (Dako Corporation, Carpinteria, CA, USA) and incubating for $20 \mathrm{~min}$ in a steamer at $100^{\circ} \mathrm{C}$. The slides were taken out of the steamer, and allowed to cool for $20 \mathrm{~min}$ in the antigen retrieval solution. After rinsing with water, avidin/biotin blocking was performed for $15 \mathrm{~min}$ each using an avidin/biotin blocking kit (SP-2001) (Vector Laboratories, Burlingame, CA, USA). Nonspecific binding sites were then blocked by incubating slides in $1 \times$ phosphate buffer saline with $0.05 \%$ Tween-20 (PBST) and $0.3 \%$ casein. Sections were then incubated with a rat monoclonal primary antibody 439-9B (Chemicon, Temecula, CA, USA) for the integrin $\beta 4$ subunit, rat IgG (negative control) 
at $5 \mu \mathrm{g} / \mathrm{ml}$, diluted in antibody diluent solution (S3022) (Dako Corporation), or antibody diluent only for $1 \mathrm{~h}$ at room temperature. After rinsing three times for $10 \mathrm{~min}$ with PBST, slides where incubated for $30 \mathrm{~min}$ with $10 \mu \mathrm{g} / \mathrm{ml}$ of a rat biotinylated secondary antibody (Vector Laboratories, Burlingame, CA, USA) in $1 \times$ DPBS and detected with an indirect streptavidin-biotin immunoperoxidase technique using Universal DakoCytomation Labeled Streptavidin-Biotin ${ }^{\circledR} 2$ System, Horseradish Peroxidase and developed with $3,3^{\prime}$-diaminobenzidine (DAB) (Dako Corporation). Sections were then counterstained with Mayer's hematoxylin, rinsed with water, dehydrated and coverslipped with Cytoseal 60 Mounting Media (Richard-Allan Scientific, Kalamazoo, MI, USA).

\section{Immunohistochemical Analysis}

Expression patterns were analyzed using standard pathological criteria and scored using a semiquantitative method based on the intensity and localization of immunostaining. Normal, pancreatic adenocarcinoma and chronic pancreatitis sample areas were scored from 0 to 3 depending on the staining intensity: $3=$ very intense staining (cytoplasmic and membranous); $2=$ moderate staining (cytoplasmic and membranous); $1=$ staining only at the basal surface interface with the basement membrane; and $0=$ no staining. PanIN lesions, PanIN 1A $(n=104)$, PanIN 1B $(n=74)$, PanIN 2 $(n=19)$ and PanIN $3 \quad(n=8)$ were identified in samples from UTMB, following the guidelines previously described, ${ }^{3}$ which are summarized on the left side of Figure 2. PanINs were identified in one case of normal pancreas, 14 cases of chronic pancreatitis and 20 cases of pancreatic adenocarcinomas. Endothelial cells and cells in the peripheral nerves served as internal positive controls; ${ }^{22,23}$ absence of staining in these cells was used as criteria to remove a sample from the study. Images were captured with a Nikon Digital Sight DS 5M color camera adapted to an upright Nikon Microscope Optiphot-2.

\section{Statistical Analysis}

Staining data were analyzed using Sigma Stat 2.03 software statistical program (Systat Software, Point Richmond, CA, USA). The nonparametric Mann-
Whitney Rank Sum Test was used to evaluate statistical significance differences between the immunohistochemical staining intensity of normal pancreas, pancreatic adenocarcinoma and chronic pancreatitis cases.

\section{Results}

\section{Integrin $\alpha 6 \beta 4$ Expression and Localization is Altered in Pancreatic Adenocarcinomas Compared to Normal Pancreas}

Integrin $\alpha 6 \beta 4$ expression is increased in several types of invasive and metastatic carcinomas including breast, colon, thyroid, gastric, bladder and squamous carcinomas and has been shown to be associated with an invasive phenotype. ${ }^{16}$ Therefore, due to the high rate of tumor dissemination associated with pancreatic adenocarcinoma, we hypothesized that integrin $\alpha 6 \beta 4$ would be overexpressed in the pancreatic cancer cells. To test this hypothesis, we performed immunohistochemistry for the integrin $\beta 4$ subunit in four cases with histological normal pancreas and 35 uninvolved normal tissues from pancreatic resections for cancer. Since the integrin $\beta 4$ subunit is known to only associate with the integrin $\alpha 6$ subunit, the staining for the $\beta 4$ subunit is indicative of integrin $\alpha 6 \beta 4$ expression. As an internal positive control, we used staining of the integrin $\beta 4$ subunit associated with endothelial cells (Figure 2b, asterisks), and Schwann cells in the peripheral nerves (Figure 1h) which have been reported to express this integrin previously. ${ }^{22,23}$ The ductal epithelial cells of these normal pancreatic tissues $(97 \%, n=39)$ showed expression of integrin $\alpha 6 \beta 4$ where it preferentially localized at the cellular interface with the basement membrane (Figures $1 \mathrm{~b}$ and $2 \mathrm{~b}$ ). Interestingly, the cells within the pancreatic acini did not display any immunoreactivity for the $\beta 4$ integrin subunit; however, cells localized at the base of the acinar epithelium stained positive for integrin $\alpha 6 \beta 4$. These cells are likely endothelial or stellate cells associated with the acini as myoepithelial cells are not understood to exist in the pancreatic acini.

In contrast, pancreatic adenocarcinoma showed overexpression of the integrin $\alpha 6 \beta 4$ in $92 \%$ (104/ 113) of the cases examined compared to normal pancreas $(P=0.002)$ or normal uninvolved tissue $(P<0.001)$ when compared to chronic pancreatitis

Figure 1 Expression of integrin $\beta 4$ in normal human pancreas and pancreatic adenocarcinomas. Tissue sections were immunostained for the integrin $\beta 4$ subunit $(\mathbf{b}, \mathbf{d}, \mathbf{f}, \mathbf{h}, \mathbf{j})$ and their respective serial sections with $\mathrm{H} \& \mathrm{E}$ (a, $\mathbf{c}, \mathbf{e}, \mathbf{g}, \mathbf{i})$. IgG as well as secondary only controls were performed and showed no immunoreactivity (data not shown). (a, b) Show representative serial sections containing normal pancreas were positive staining is found in the basal surface at the interface with the basement membrane of epithelial cells in the ductal component of the pancreas ( $\times 400$ original magnification). (c-j) Show representative areas of cancers where expression of integrin $\beta 4$ is seen upregulated when compared to normal pancreas and it localized to the basal, apical and lateral sides of the membrane as well as within the cytoplasm of cancer cells $(\mathbf{c}-\mathbf{f}, \times 100$; $\mathbf{g}$ and $\mathbf{h}, \times 200 ; \mathbf{i}$ and $\mathbf{j}, \times 40)$. (g, h) Show pancreatic carcinoma with perineural invasion in which Schwann cells (also known to express integrin $\alpha 6 \beta 4$ and serve as an internal positive control) and pancreatic cancer cells stained for the integrin $\beta 4$ subunit. (i, j) Show pancreatic adenocarcinoma lymph node metastasis. Insets in (b), (f) and (h) show the difference in expression and localization of the integrin $\alpha 6 \beta 4$ between normal and pancreatic cancer cells. Arrows (d and $\mathbf{f}$ ) show invading cancer cells, identified by the integrin $\beta 4$ staining. 
samples. Notably, the localization of the $\alpha 6 \beta 4$ integrin in pancreatic carcinoma cells was no longer restricted to the basal surface, but also extended to the apical and lateral sides of the cell membrane as well as within the cytoplasm (Figures 1d, f, h, j and 2j). A similar pattern of expression was noted in pancreatic cancer that had invaded into the peripheral nerves (Figure $1 \mathrm{~h}$ ) or lymph nodes (Figure 1j). Of the 20 pancreatic adenocarcinomas for which we had ample tissue to analyze (UTMB
H\&E
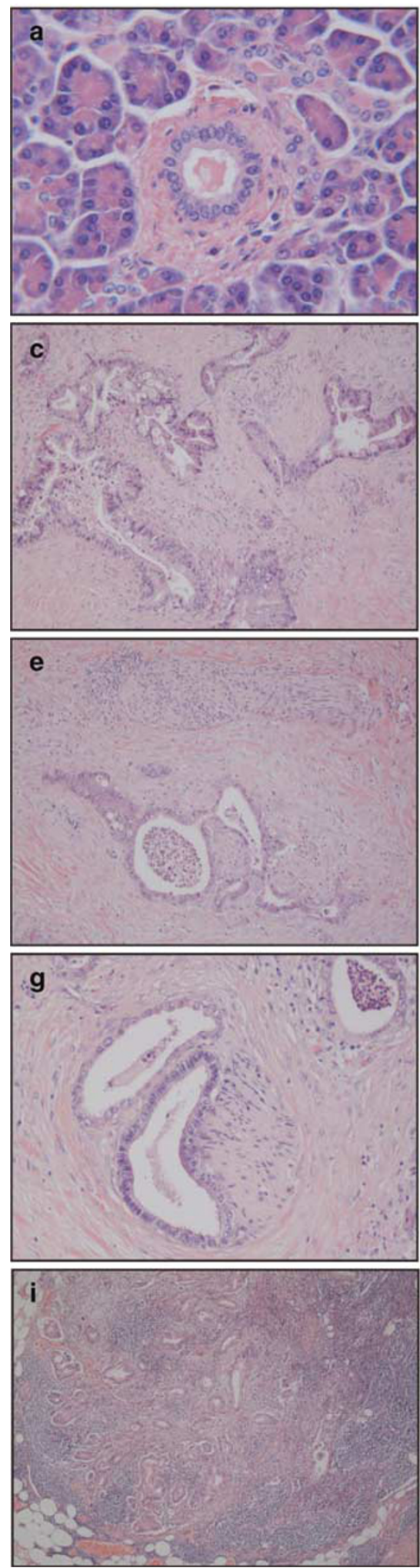

\section{Integrin $\beta 4$}
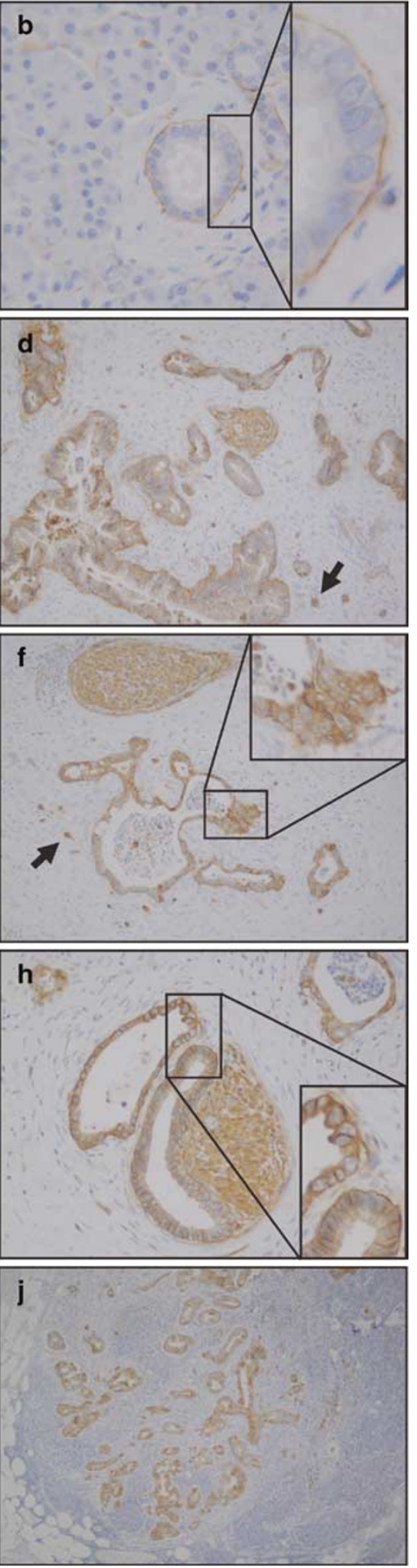
H\&E

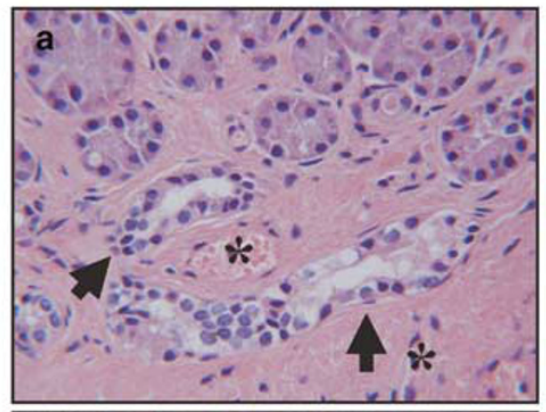

Normal duct

- single cell layer

- low cuboidal

PanIN-1A/1B

- elongated cells

- mucin

- papillary growth

\section{PanIN-2}

- early nuclear abnormalities

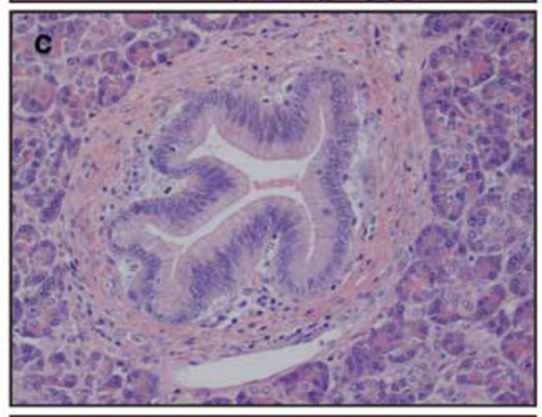

\section{PanIN-3}

- luminal budding

- nuclear atypia

- mitosis

\section{Carcinoma}

- invasion

- desmoplasia
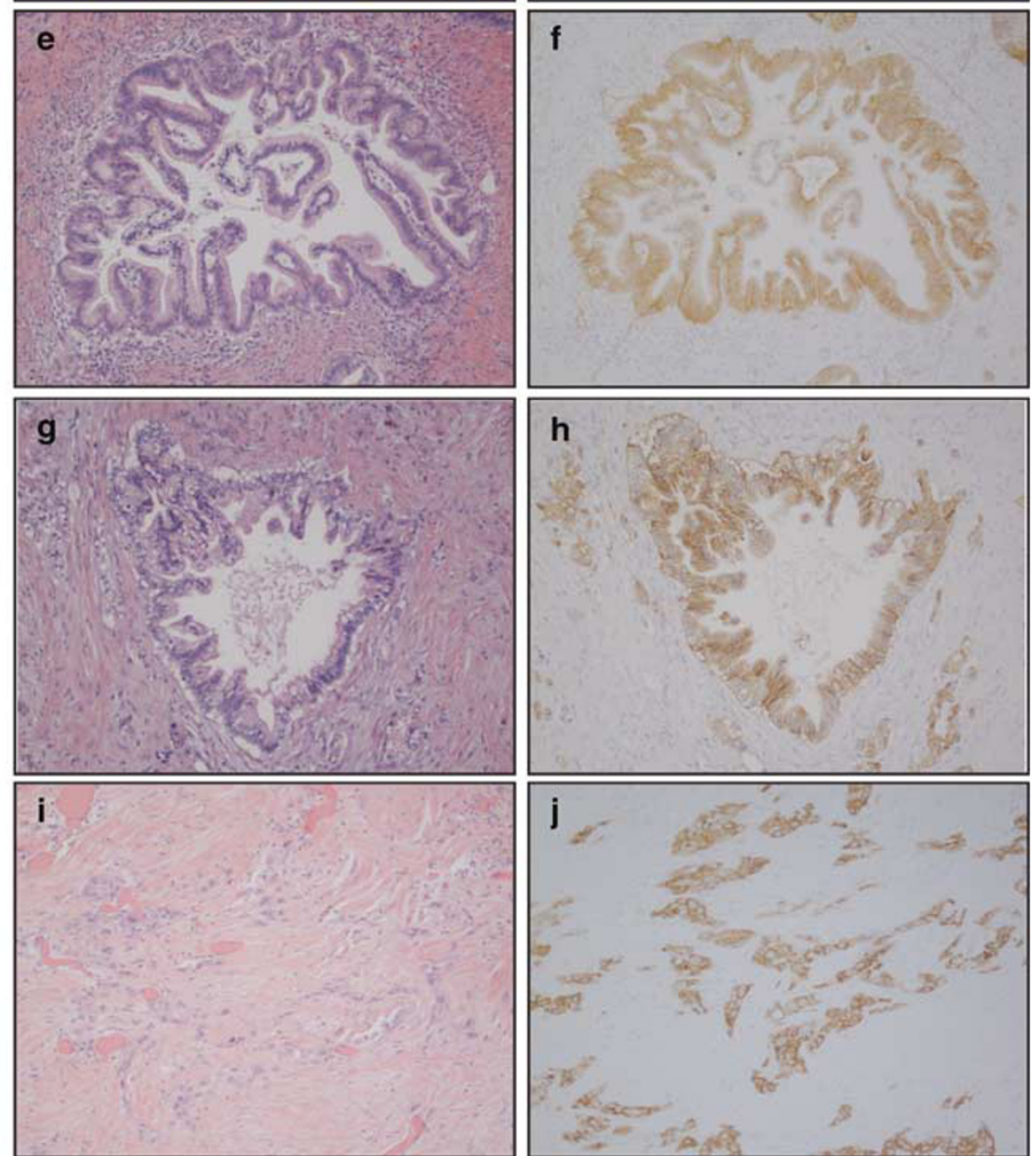

Figure 2 Expression of integrin $\beta 4$ in the PanIN progression model. Tissue sections containing representative normal pancreas (a and b), PanIN lesions ( $\mathbf{c}-\mathbf{h}$ ) and carcinoma (i and $\mathbf{j})$ were immunostained for the integrin $\beta 4$ subunit $(\mathbf{b}, \mathbf{d}, \mathbf{f}, \mathbf{h}, \mathbf{j})$ and their respective serial sections with H\&E $(\mathbf{a}, \mathbf{c}, \mathbf{e}, \mathbf{g}, \mathbf{i})$. As in Figure 1a and b show normal pancreatic ducts where the staining for integrin $\beta 4$ subunit is only seen surrounding the basement membrane of the epithelial ducts (arrows) $(\times 400)$. Endothelial cells serve as an internal control for the stain quality (asterisks). (c-h) Show representative PanIN lesions as describe in the left side of the figure which summarizes the criteria used in reference ${ }^{3}(\mathbf{c}-\mathbf{d}, \times 200 ; \mathbf{e}-\mathbf{h}, \times 100)$. (g, h) Shows invasive carcinoma adjacent to PanIN-3. (i, j) Show a representative sample of a patient specimen with invasive pancreatic carcinoma $(\times 100)$. 
cases), six cases demonstrated perineural invasion and six cases had lymph node metastasis. In all of these cases, tumor cells displayed overexpression and altered localization of the $\beta 4$ integrin subunit; cytokeratin staining was performed to confirm the epithelial nature of cells (data not shown). Insets in Figure $1 \mathrm{~b}, \mathrm{f}$, and h demonstrate the clear distinction in localization and expression of integrin $\alpha 6 \beta 4$ between normal pancreas and pancreatic adenocarcinoma cells. In addition, single invading cells can be distinguished in the stromal environment by their intense integrin $\beta 4$ subunit staining (Figure $1 \mathrm{~d}$ and $\mathrm{f}$, arrows), which are difficult to identify by H\&E staining. Cytokeratin staining was performed on serial sections to confirm the epithelial nature of cells invading the stromal environment of the tissues (data not shown). For all cases, IgG and/or secondary biotinylated antibody only controls were performed and no immunoreactivity was observed (data not shown).

The summary of patients analyzed is listed in Table 1. The majority of patients with pancreatic adenocarcinoma presented with high staining intensity of integrin $\alpha 6 \beta 4$. In contrast, normal or normal uninvolved tissue displayed low (basal surface only) or no staining. Notably, we found that the antibody used in this study was sensitive to the antigen retrieval method used which is affected by the way tissues are fixed after resection. For this reason, several specimens were not included in the study due to an absence of staining in control cells within the specimen. Most cases that showed no staining or moderate expression levels of the integrin $\beta 4$ subunit were from the tissue microarrays for which there was no internal control to assess the quality of the tissue. Therefore, the percentage of pancreatic adenocarcinomas with high $\alpha 6 \beta 4$ expression may be higher considering that $100 \% \quad(n=20)$ of the pancreatic adenocarcinomas from our institution demonstrated intense staining. Overall, our results strongly suggest that overexpression and redistribution of the integrin $\alpha 6 \beta 4$ is a prominent feature of pancreatic adenocarcinoma.
Integrin $\alpha 6 \beta 4$ Expression is Elevated and Altered in PanIN Lesions

PanIN lesions are considered precursors of pancreatic adenocarcinomas. ${ }^{3}$ Therefore, we analyzed the expression pattern of integrin $\alpha 6 \beta 4$ in PanIN lesions to determine if this integrin could play a role in pancreatic cancer development. Hence, we identified PanINs of grades 1-3 in most of the pancreatic adenocarcinomas and chronic pancreatitis tissues analyzed from our institution based on established criteria. $^{3}$ These criteria are summarized on the left side of Figure 2. Here, we found that lesions as early as PanIN 1A showed moderate to high expression as well as altered localization of the integrin $\alpha 6 \beta 4$, similar to the pattern noted in pancreatic adenocarcinomas. Interestingly, this pattern of expression was maintained throughout the established PanIN progression model (Figure 2a-j and Table 2). In addition, we were able to identify several transitional lesions in which cells displaying a columnar morphology and increased mucin characteristic of PanIN 1A/1B were adjacent to low cuboidal epithelium of a normal duct. These transition regions reveal that the dramatic upregulation and altered localization of integrin $\beta 4$ subunit are associated with the first morphological changes that characterize PanIN lesions (Figure 3). Therefore, these data suggest that increased expression and redistribution of the integrin $\alpha 6 \beta 4$ in pancreatic adenocarcinomas is an early and prominent event in the development of pancreatic cancers that is associated with the first discernable step in pancreatic tumor progression.

\section{Integrin $\alpha 6 \beta 4$ Expression in Chronic Pancreatitis is Similar to Normal Pancreatic Ducts}

Chronic pancreatitis is an inflammatory disease of the pancreas that has similar histological characteristics as pancreatic adenocarcinoma, which can

Table 1 Integrin $\beta 4$ subunit expression in pancreatic tissues

\begin{tabular}{|c|c|c|c|c|c|}
\hline \multirow[t]{2}{*}{ Diagnosis } & \multicolumn{4}{|c|}{ Staining intensity ${ }^{\mathrm{a}}$ percentage of cases } & \\
\hline & 0 & 1 & 2 & 3 & \\
\hline Normal pancreas $(n=4)$ & & 100 & & & \\
\hline Chronic pancreatitis $(n=14)$ & & 93 & 7 & & $P=0.871$ vs normal \\
\hline Pancreatic adenocarcinoma $(n=113)$ & 2 & 6 & 29 & 63 & $\begin{array}{l}P=0.002^{*} \text { vs normal } \\
P<0.001^{*} \text { vs chronic pancreatitis }\end{array}$ \\
\hline $\begin{array}{l}\text { Normal uninvolved tissue of pancreatic } \\
\text { adenocarcinoma cases }(n=35)^{\mathrm{b}}\end{array}$ & 3 & 97 & & & \\
\hline $\begin{array}{l}\text { Chronic pancreatitis area within pancreatic } \\
\text { adenocarcinoma cases }(n=15)^{\mathrm{b}}\end{array}$ & & 93 & 7 & & \\
\hline
\end{tabular}

\footnotetext{
${ }^{a}$ Staining intensity criteria are described in the Materials and methods section.

${ }^{b}$ Pancreatic adenocarcinoma cases that had normal uninvolved areas and/or chronic pancreatitis areas.

*Statistical significance using the Mann-Whitney rank sum test.
} 
Table 2 Integrin $\beta 4$ subunit expression in pancreatic intraepithelial neoplasia lesions

\begin{tabular}{|c|c|c|c|c|}
\hline \multirow[t]{2}{*}{ Type of lesion } & \multirow[t]{2}{*}{ Number of lesions identified } & \multicolumn{3}{|c|}{ Staining intensity ${ }^{\mathrm{a}}$ percentage of lesions } \\
\hline & & 1 & 2 & 3 \\
\hline PanIN 1A & 104 & & 25 & 75 \\
\hline PanIN 1B & 74 & & 3 & 97 \\
\hline PanIN 2 & 19 & & & 100 \\
\hline PanIN 3 & 8 & & & 100 \\
\hline
\end{tabular}

${ }^{\mathrm{a}}$ Staining intensity criteria are described in the Materials and methods section.

Only cases from UTMB were included in this analysis, due to the ample tissue available in these samples.

\section{H\&E}
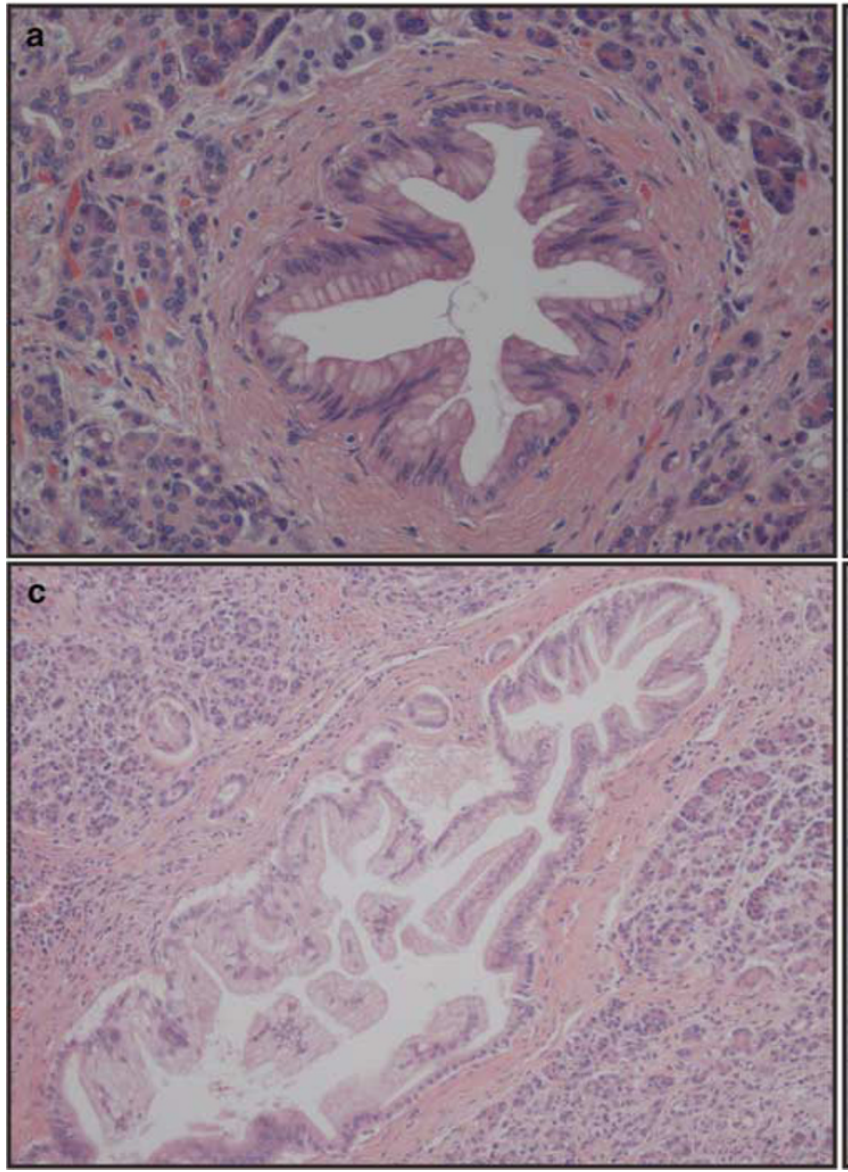

\section{Integrin $\beta 4$}

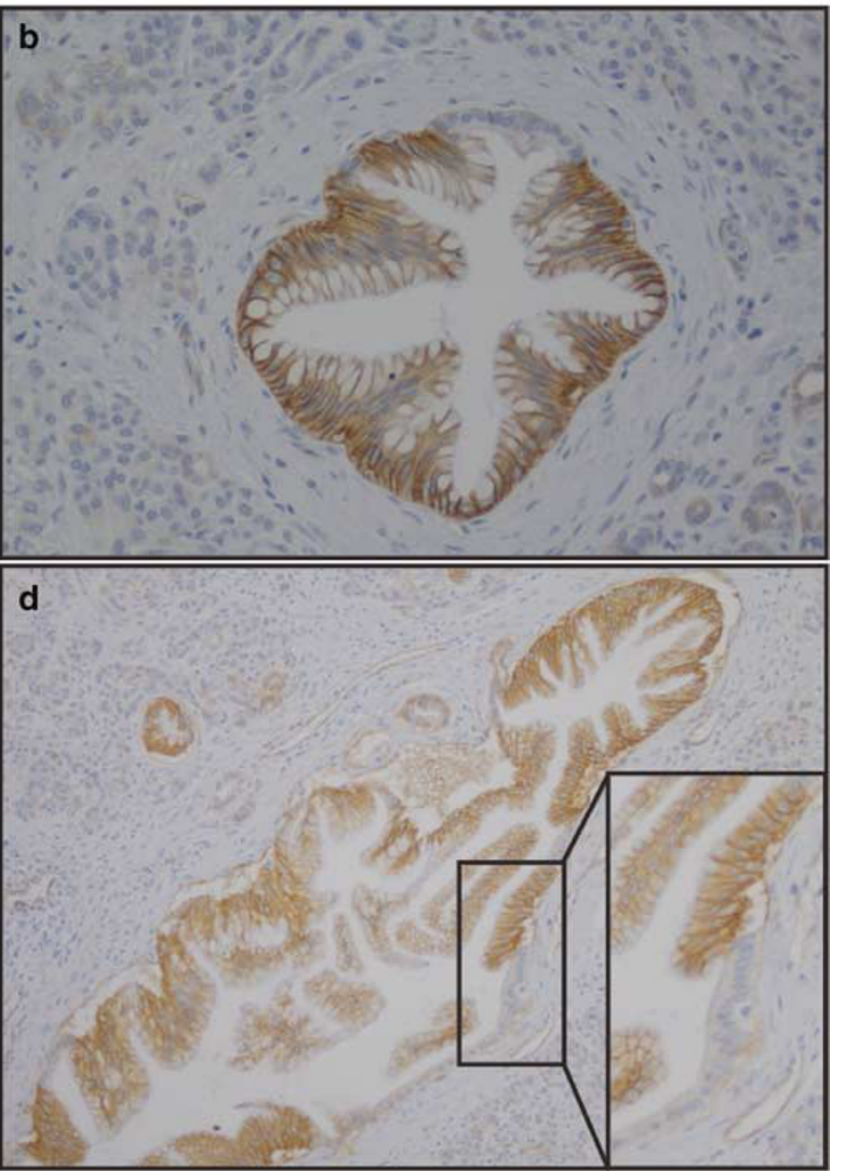

Figure 3 Expression of integrin $\beta 4$ in PanIN lesion showing residual normal duct. Immunostaining for the integrin $\beta 4$ subunit (b and $\mathbf{d}$ ) and respective serial section stained with H\&E (a and c). Panels (a) and (b) show a PanIN-1A/1B lesion showing an area of residual normal low cuboidal epithelium $(\times 200)$. Transitional area in (b) shows the dramatic redistribution and overexpression of the integrin $\beta 4$ subunit in early PanIN lesion when compare to the normal cells. Panels (c) and (d) show an example of a PanIN-2 lesion that also show areas with residual normal low cuboidal epithelium. Inset in panel (d) show the dramatic change in the integrin $\beta 4$ subunit expression and localization as the epithelium changes from normal to preneoplastic $(\times 100)$.

make the distinction of the two diseases challenging. To investigate whether integrin $\beta 4$ subunit staining could distinguish pancreatic cancer from chronic pancreatitis, tissue sections from 14 patients with the final diagnosis of chronic pancreatitis were analyzed for integrin $\alpha 6 \beta 4$ expression. In addition, areas of chronic pancreatitis identified within pancreatic adenocarcinoma samples $(n=15)$ were analyzed. As shown in Figure $4 \mathrm{~b}$ and $\mathrm{d}$, integrin $\beta 4$ staining in chronic pancreatitis samples, 93\% (13/ 14) resembles the staining pattern of normal pancreatic ducts $(P=0.871)$ where the integrin $\alpha 6 \beta 4$ 


\section{H\&E}
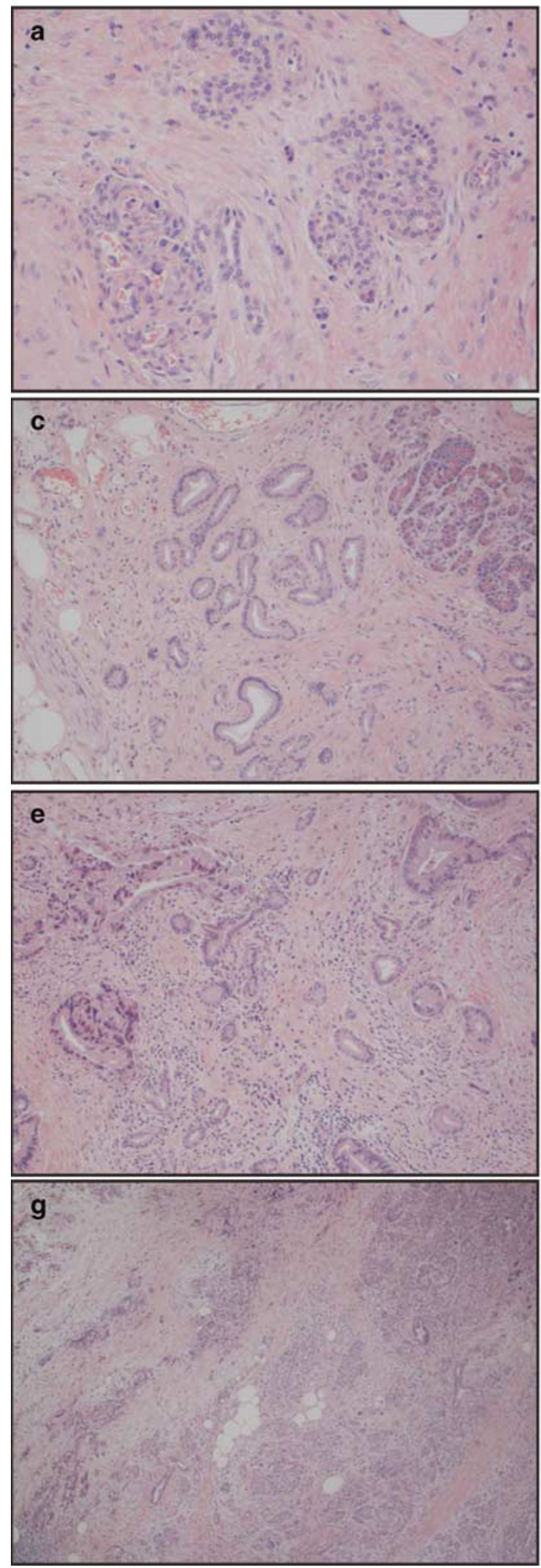

\section{Integrin $\beta 4$}
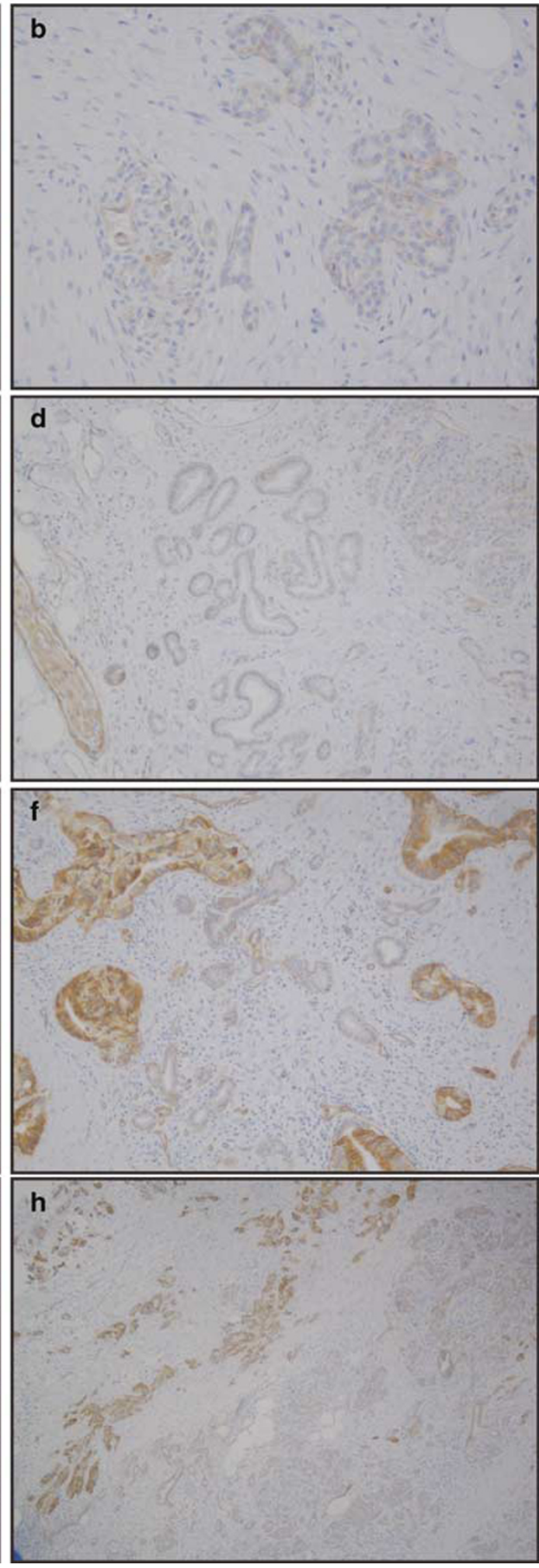

Figure 4 Expression of integrin $\beta 4$ in a patient with pancreatic cancer and pancreatitis. Tissue sections of a patient diagnosed with chronic pancreatitis were immunostained for the integrin $\beta 4$ subunit (b) and the respective serial section with H\&E (a). (a and b, $\times 200)$ Show an area of chronic pancreatitis for which the integrin $\beta 4$ staining resembles the normal pancreas. (c, h) Show sections of cases that presented with both pancreatic adenocarcinoma and chronic pancreatitis. (c and $\mathbf{d}, \times 100)$ Show chronic pancreatitis area only where the staining pattern resembles that of normal pancreas. (e and $\mathbf{f}, \times 100$ and $\mathbf{g}$ and $\mathbf{h}, \times 40$ ) Show an area of cancer and chronic pancreatitis in the same area at different magnifications. 
staining is restricted to the basal plasma membrane (Figures $1 \mathrm{~b}$ and $2 \mathrm{~b}$ ). The quantification of these observations is shown in Table 1. Interestingly, when pancreatic adenocarcinoma and chronic pancreatitis were found in the same patient specimen, the dramatic overexpression and altered localization of integrin $\alpha 6 \beta 4$ present within the cancer nicely distinguished the cancer from chronic pancreatitis (Figure $4 \mathrm{f}$ and $\mathrm{h}$ ). These results demonstrate that the integrin $\alpha 6 \beta 4$ expression pattern noted in pancreatic adenocarcinomas is consistently and dramatically different from chronic pancreatitis.

\section{Discussion}

Pancreatic adenocarcinomas are highly aggressive and metastatic cancers that are thought to arise from noninvasive neoplastic precursor PanIN lesions. ${ }^{5}$ A novel finding in our study is that the upregulation and altered localization of the integrin $\alpha 6 \beta 4$ occurs early in pancreatic adenocarcinoma tumor progression (ie PanIN 1A lesions). Here, we find that integrin $\alpha 6 \beta 4$ is overexpressed in all 205 of the PanIN lesions identified. Interestingly, we were able to identify PanIN lesions juxtapositioned to residual normal low cuboidal epithelium (Figure 3). The changes in $\alpha 6 \beta 4$ integrin expression and distribution were noted at the interface where the abrupt transformation between normal and preneoplastic lesions occurs. This change within the transitional areas provides evidence that this integrin is overexpressed at an early stage in pancreatic adenocarcinoma development and it may be upregulated as a result of, or directly contribute to, the initiation of PanIN lesions that may eventually develop into an invasive carcinoma. Interestingly, another major finding here is that the $\alpha 6 \beta 4$ integrin expression and altered localization associated with PanIN lesions is sustained during pancreatic cancer development and is associated with pancreatic adenocarcinomas. In this study, we show that the integrin $\alpha 6 \beta 4$ is dramatically overexpressed in most of the pancreatic adenocarcinoma samples analyzed $(92 \%, 104 / 113)$ when compared to normal pancreas and chronic pancreatitis samples. Therefore, our data suggest that the integrin $\alpha 6 \beta 4$ is likely involved in cancer development due to the prevalence of its overexpression in the earliest preneoplastic lesions and its persistence through tumor progression.

Multiple studies have correlated integrin $\alpha 6 \beta 4$ with tumor progression, predominantly with the later stages of progression where cells acquire the ability to invade and metastasize. In bladder cancers, the integrin $\alpha 6 \beta 4$ staining pattern correlated with the histological stage and grade of tumors ${ }^{24}$ while in colorectal cancers, the integrin $\alpha 6 \beta 4$ expression was found to be increased predominantly in less differentiated cancers. ${ }^{25}$ Integrin $\alpha 6 \beta 4$ also is expressed at the invasive fronts in association with its ligands laminin-1 and laminin-5 in gastric cancers ${ }^{26}$ where it is suggested to be involved in the invasion process. In pancreatic cancer, prior studies using gene microarray analysis and immunohistochemistry demonstrated that the $\beta 4$ integrin subunit was upregulated when compared to normal pancreas and pancreatitis tissues ${ }^{10,11,13}$ as well as associated with cases that presented with lymph node metastasis. ${ }^{12}$ Furthermore, the potential role of the $\alpha 6 \beta 4$ integrin in metastasis is supported by studies in papillary thyroid carcinoma which suggested that the increased expression of the integrin $\beta 4$ subunit in cancer lesions could play a role in the development of lymph node metastasis. ${ }^{27}$ Certainly, the in vivo data coupled with extensive in vitro data suggest that the $\alpha 6 \beta 4$ is associated with the invasiveness and metastatic potential of late stage cancers. ${ }^{16,24-27}$ In our study, the six pancreatic adenocarcinoma cases that were associated with lymph node metastasis displayed overexpression of the integrin $\beta 4$ subunit in cancer cells (Figure 1i-j). When pancreatic cells were found invading the peripheral nerves, the invading cancer cells also overexpressed integrin $\alpha 6 \beta 4$. We find that the integrin $\alpha 6 \beta 4$ is upregulated and redistributed early during pancreatic tumor progression, and persists through progression to lymph node metastasis. Therefore, our findings suggest that, unlike carcinomas of the breast, bladder, colon, stomach and thyroid, increased expression of integrin $\alpha 6 \beta 4$ in pancreatic adenocarcinomas occurs early rather than later in progression.

Interestingly, the expression pattern of integrin $\alpha 6 \beta 4$ in most of the pancreatic adenocarcinomas studied changes from basal membrane localization in normal pancreatic ductal cells to the cytoplasm (where it now localizes), and the apical and lateral regions of the cell membrane. This pattern of expression is similar to that noted in squamous cell carcinomas and adenocarcinomas of the lung, where the integrin $\alpha 6 \beta 4$ was found to stain the plasma membrane and the cytoplasm of tumor cells. ${ }^{28}$ The cytoplasmic staining seen in most of the cancers that overexpressed the $\beta 4$ integrin subunit is likely due to the excess synthesis of this chain since there are no known signaling functions associated with cytoplasmic pools of integrin subunits. Integrins require dimerization of the $\alpha$ and $\beta$ subunits to be transported to the plasma membrane where they function as extracellular matrix receptors and signal transducers. Therefore, it is likely that the $\beta 4$ subunit is synthesized in excess and resides in the endoplasmic reticulum until paired with newly synthesized $\alpha 6$ subunit prior to transport to the plasma membrane. These observations are in good agreement with early studies on integrins and pancreatic carcinomas that reported the $\alpha 6$ integrin subunit to be diffusely distributed on the surface of carcinoma cells and to be present at the RNA level in pancreatic cancer cell lines and pancreatic tumor extracts. ${ }^{29,30}$ Therefore, it is the plasma membrane- 
associated integrins that are expected to function in adhesion and signaling.

How the integrin $\alpha 6 \beta 4$ contributes to pancreatic adenocarcinoma development could be explained by the ability of integrin $\alpha 6 \beta 4$ to interact with and facilitate signaling from several molecules understood to be upregulated in pancreatic cancers. Evidence shows that cooperation between the $\alpha 6 \beta 4$ integrin and activated forms of Ras can lead to the formation of human squamous cell carcinomas. ${ }^{31}$ Importantly, activating K-Ras mutations are present in virtually all pancreatic adenocarcinomas and are suggested to play a role in tumor initiation due to its activation in early PanIN lesions. ${ }^{32}$ Moreover, recent mouse and rat models have shown the importance of Ras mutations in pancreatic adenocarcinoma development. ${ }^{33,34}$ The hepatocyte growth factor receptor, c-Met, is another molecule of interest found to be upregulated in early PanIN lesions and pancreatic adenocarcinomas. ${ }^{35,36} \mathrm{c}$-Met is known to cooperate with integrin $\alpha 6 \beta 4$ to enhance the migratory and invasive potential of cancer cells in vitro. ${ }^{37,38}$ Furthermore, the tumorigenic potential of c-Met in cancer cells in vitro has been attributed to its cooperation with the integrin $\alpha 6 \beta 4 .^{39}$ The integrin $\alpha 6 \beta 4$ has also been shown to promote the signaling of transcription factors such as NFAT-1 in carcinoma cells. ${ }^{40}$ NFAT-1 was found to be amplified in pancreatic cancers ${ }^{41}$ which could lead to the regulation of proinvasive genes, such as seen in breast carcinomas with NFAT-mediated upregulation of autotaxin. ${ }^{42}$ Furthermore, NFAT-1 can also induce the expression of cyclooxygenase $-2^{43}$ which is known to be overexpressed in pancreatic tumors. ${ }^{44}$ The association of the $\alpha 6 \beta 4$ integrin and known cooperating oncogenes such as Ras and c-Met and regulation of transcription factors, such as NFAT-1 in early tumor precursor lesions, suggests that integrin $\alpha 6 \beta 4$ could cooperate and/or activate these factors to drive the development and progression of pancreatic adenocarcinomas and contribute to the invasive and metastatic phenotype of these cancers. Therefore, we suggest that early expression of $\alpha 6 \beta 4$ in PanIN lesions can predispose pancreatic adenocarcinomas to become among the most invasive and metastatic cancers. Certainly, future studies are needed to determine the cooperation of integrin $\alpha 6 \beta 4$ with these factors and to elucidate how they facilitate the invasion and metastasis of pancreatic cancer cells.

A common challenge for clinicians with a patient that has a pancreatic mass is to determine if it is pancreatic cancer or chronic pancreatitis, as the two diseases might present with similar symptoms and histological characteristics. This distinction is crucial for treatment and management of each disease. Fine needle aspiration cytology (FNAC) obtained during endoscopic ultrasonography (EUS) has been employed for diagnosing pancreatic masses; however, it is useful as a positive predictive but not as a negative predictor since cancer could be missed during sampling. ${ }^{45}$ Even with FNAC, it can be difficult to determine if a lesion is cancer, preneoplastic or pancreatitis. Here, we show that 93\% (13/ 14) of the chronic pancreatitis samples and $100 \%$ $(15 / 15)$ of the chronic pancreatitis areas found within the patients with pancreatic adenocarcinomas resembled the $\alpha 6 \beta 4$ integrin expression pattern of normal pancreas. Moreover, when chronic pancreatitis was present in pancreatic adenocarcinoma samples, we were able to distinguish the areas of pancreatic cancer easily as the integrin $\alpha 6 \beta 4$ staining pattern highlighted the areas of cancer in a particular pancreatic tissue, even when it was not apparent with H\&E staining. We suggest that the staining pattern of integrin $\alpha 6 \beta 4$, in conjunction with other markers and histological characteristics, could aid in distinguishing pancreatic cancer from chronic pancreatitis as well as detect early lesions of pancreatic cancer.

In summary, this study shows that the integrin $\alpha 6 \beta 4$ is upregulated in pancreatic cancers and that its localization is altered, where it is no longer restricted to the basal surface and can be found in the cytosol, and on the basal, lateral and apical sides of the plasma membrane. These events occur early in the progression of pancreatic cancer development starting at the PanIN-1A stage. Furthermore, this study shows that the altered staining pattern of integrin $\alpha 6 \beta 4$ in pancreatic adenocarcinoma tissues can help identified cancer areas in the background of chronic pancreatitis. Our study is in agreement with mounting evidence that the integrin $\alpha 6 \beta 4$ plays a major role in pancreatic adenocarcinomas. Certainly, further studies are needed to understand the mechanisms involved in the induction of the integrin $\beta 4$ subunit expression and redistribution, as well as how it contributes to cancer progression.

\section{Acknowledgements}

We gratefully acknowledge Linda E Muehlberger for her help in troubleshooting the immunohistochemistry method, Dr William Nealon and Dr Jingwu Xie for helpful discussions, Karen Martin for graphics assistance and Dr James Brady for aid with statistical analysis. This work was supported by the National Institutes of Health Grants R21-CA102125 (KLO) and F31 CA106201 (ZCM).

\section{References}

1 Jemal A, Siegel R, Ward E, et al. Cancer statistics, 2006. CA Cancer J Clin 2006;56:106-130.

2 Li D, Xie K, Wolff R, et al. Pancreatic cancer. Lancet 2004;363:1049-1057.

3 Hruban RH, Adsay NV, Albores-Saavedra J, et al. Pancreatic intraepithelial neoplasia: a new nomenclature and classification system for pancreatic duct lesions. Am J Surg Pathol 2001;25:579-586. 
4 Hruban RH, Goggins M, Parsons J, et al. Progression model for pancreatic cancer. Clin Cancer Res 2000;6: 2969-2972.

5 Kloppel G, Luttges J. The pathology of ductal-type pancreatic carcinomas and pancreatic intraepithelial neoplasia: insights for clinicians. Curr Gastroenterol Rep 2004;6:111-118.

6 Volkholz H, Stolte M, Becker V. Epithelial dysplasias in chronic pancreatitis. Virchows Arch A Pathol Anat Histol 1982;396:331-349.

7 Malka D, Hammel P, Maire F, et al. Risk of pancreatic adenocarcinoma in chronic pancreatitis. Gut 2002;51: 849-852.

8 Lowenfels AB, Maisonneuve P, Cavallini G, et al. Pancreatitis and the risk of pancreatic cancer. International Pancreatitis Study Group. N Engl J Med 1993; 328:1433-1437.

9 Taylor B. Carcinoma of the head of the pancreas versus chronic pancreatitis: diagnostic dilemma with significant consequences. World J Surg 2003;27:1249-1257.

10 Logsdon CD, Simeone DM, Binkley C, et al. Molecular profiling of pancreatic adenocarcinoma and chronic pancreatitis identifies multiple genes differentially regulated in pancreatic cancer. Cancer Res 2003;63: 2649-2657.

11 Crnogorac-Jurcevic T, Missiaglia E, Blaveri E, et al. Molecular alterations in pancreatic carcinoma: expression profiling shows that dysregulated expression of S100 genes is highly prevalent. J Pathol 2003;201: 63-74.

12 Nakamura T, Furukawa Y, Nakagawa H, et al. Genomewide cDNA microarray analysis of gene expression profiles in pancreatic cancers using populations of tumor cells and normal ductal epithelial cells selected for purity by laser microdissection. Oncogene 2004; 23:2385-2400.

13 Gleason B, Adley B, Rao MS, et al. Immunohistochemical detection of the beta4 integrin subunit in pancreatic adenocarcinoma. J Histochem Cytochem 2005;53: 799-801.

14 Hynes RO. Integrins: bidirectional, allosteric signaling machines. Cell 2002;110:673-687.

15 Guo W, Giancotti FG. Integrin signalling during tumour progression. Nat Rev Mol Cell Biol 2004;5:816-826.

16 Mercurio AM, Rabinovitz I. Towards a mechanistic understanding of tumor invasion-lessons from the alpha6beta 4 integrin. Semin Cancer Biol 2001;11: 129-141.

17 Grossman HB, Lee C, Bromberg J, et al. Expression of the alpha6beta4 integrin provides prognostic information in bladder cancer. Oncol Rep 2000;7:13-16.

18 Hemler ME, Crouse C, Sonnenberg A. Association of the VLA alpha 6 subunit with a novel protein. A possible alternative to the common VLA beta 1 subunit on certain cell lines. J Biol Chem 1989;264:6529-6535.

19 Borradori L, Sonnenberg A. Structure and function of hemidesmosomes: more than simple adhesion complexes. J Invest Dermatol 1999;112:411-418.

20 Rabinovitz I, Toker A, Mercurio AM. Protein kinase Cdependent mobilization of the alpha6beta4 integrin from hemidesmosomes and its association with actinrich cell protrusions drive the chemotactic migration of carcinoma cells. J Cell Biol 1999;146:1147-1160.

21 Lipscomb EA, Mercurio AM. Mobilization and activation of a signaling competent alpha6beta4 integrin underlies its contribution to carcinoma progression. Cancer Metast Rev 2005;24:413-423.
22 Hiran TS, Mazurkiewicz JE, Kreienberg P, et al. Endothelial expression of the alpha6beta4 integrin is negatively regulated during angiogenesis. J Cell Sci 2003;116:3771-3781.

23 Feltri ML, Scherer SS, Nemni R, et al. Beta 4 integrin expression in myelinating Schwann cells is polarized, developmentally regulated and axonally dependent. Development 1994;120:1287-1301.

24 Mialhe A, Louis J, Pasquier D, et al. Expression of three cell adhesion molecules in bladder carcinomas: correlation with pathological features. Anal Cell Pathol 1997;13:125-136.

25 Falcioni R, Turchi V, Vittulo $\mathrm{P}$, et al. Integrin beta4 expression in colorectal cancer. Int J Oncol 1994;5: 573-578.

26 Tani T, Karttunen T, Kiviluoto T, et al. Alpha 6 beta 4 integrin and newly deposited laminin-1 and laminin-5 form the adhesion mechanism of gastric carcinoma. Continuous expression of laminins but not that of collagen VII is preserved in invasive parts of the carcinomas: implications for acquisition of the invading phenotype. Am J Pathol 1996;149:781-793.

27 Kitajiri S, Hosaka N, Hiraumi $\mathrm{H}$, et al. Increased expression of integrin beta-4 in papillary thyroid carcinoma with gross lymph node metastasis. Pathol Int 2002;52:438-441.

28 Mariani Costantini R, Falcioni $\mathrm{R}$, Battista $\mathrm{P}$, et al. Integrin (alpha 6/beta 4) expression in human lung cancer as monitored by specific monoclonal antibodies. Cancer Res 1990;50:6107-6112.

29 Weinel RJ, Rosendahl A, Pinschmidt E, et al. The alpha 6 -integrin receptor in pancreatic carcinoma. Gastroenterology 1995;108:523-532.

30 Lohr M, Trautmann B, Gottler M, et al. Expression and function of receptors for extracellular matrix proteins in human ductal adenocarcinomas of the pancreas. Pancreas 1996;12:248-259.

31 Dajee M, Lazarov M, Zhang JY, et al. NF-kappaB blockade and oncogenic Ras trigger invasive human epidermal neoplasia. Nature 2003;421:639-643.

32 Moskaluk CA, Hruban RH, Kern SE. p16 and K-ras gene mutations in the intraductal precursors of human pancreatic adenocarcinoma. Cancer Res 1997;57: 2140-2143.

33 Hingorani SR, Petricoin EF, Maitra A, et al. Preinvasive and invasive ductal pancreatic cancer and its early detection in the mouse. Cancer Cell 2003;4: $437-450$.

34 Ueda S, Takasuka N, Takeshita F, et al. Ductal origin of pancreatic adenocarcinomas induced by conditional activation of a human Ha-ras oncogene in rat pancreas. Carcinogenesis 2006;27:2497-2510.

$35 \mathrm{Yu}$ J, Ohuchida K, Mizumoto K, et al. Overexpression of c-met in the early stage of pancreatic carcinogenesis; altered expression is not sufficient for progression from chronic pancreatitis to pancreatic cancer. World J Gastroenterol 2006;12:3878-3882.

36 Di Renzo MF, Poulsom R, Olivero M, et al. Expression of the Met/hepatocyte growth factor receptor in human pancreatic cancer. Cancer Res 1995;55: 1129-1138.

37 Chung J, Yoon SO, Lipscomb EA, et al. The Met receptor and alpha 6 beta 4 integrin can function independently to promote carcinoma invasion. J Biol Chem 2004;279:32287-32293 [E-pub May 25, 2004].

38 Trusolino L, Bertotti A, Comoglio PM. A signaling adapter function for alpha6beta4 integrin in the 
control of HGF-dependent invasive growth. Cell 2001;107:643-654.

39 Bertotti A, Comoglio PM, Trusolino L. Beta4 integrin is a transforming molecule that unleashes Met tyrosine kinase tumorigenesis. Cancer Res 2005;65:10674-10679.

40 Jauliac S, Lopez-Rodriguez C, Shaw LM, et al. The role of NFAT transcription factors in integrin-mediated carcinoma invasion. Nat Cell Biol 2002;4:540-544.

41 Holzmann K, Kohlhammer H, Schwaenen C, et al. Genomic DNA-chip hybridization reveals a higher incidence of genomic amplifications in pancreatic cancer than conventional comparative genomic hybridization and leads to the identification of novel candidate genes. Cancer Res 2004;64:4428-4433.
42 Chen M, O’Connor KL. Integrin alpha6beta4 promotes expression of autotaxin/ENPP2 autocrine motility factor in breast carcinoma cells. Oncogene 2005;24: 5125-5130.

43 Yiu GK, Toker A. NFAT induces breast cancer cell invasion by promoting the induction of cyclooxygenase-2. J Biol Chem 2006;281:12210-12217.

44 Okami J, Yamamoto H, Fujiwara Y, et al. Overexpression of cyclooxygenase-2 in carcinoma of the pancreas. Clin Cancer Res 1999;5:2018-2024.

45 Balthazar EJ. Pancreatitis associated with pancreatic carcinoma. Preoperative diagnosis: role of CT imaging in detection and evaluation. Pancreatology 2005;5: 330-344. 